\title{
Argumentativ überwunden, aber nicht überzeugt? Zur Wirksamkeit der Sokratischen Elenktik in Platons Gorgias
}

Bernhard Kaiser (Dresden University of Technology)

\section{Overcome by Arguments, but not Convinced? On the Efficacy of the Socratic Method in Plato's Gorgias}

\begin{abstract}
The Gorgias is frequently read as a dialogue in which Socrates fails to convince especially his last interlocutor Callicles to adopt a philosophical way of life. Instead, Callicles becomes increasingly indignant about the repeated refutation of his personal convictions and eventually refuses to participate in the conversation any longer. In general scholars explain this result by Callicles' recalcitrance. It is supposed that the dialogue illustrates the impossibility of persuasion if an interlocutor refuses to cooperate - which would imply certain limitations of the Socratic elenchus. In contrast, this paper demonstrates that Socrates indeed achieves his aim of shaking Callicles' faith. By decoding the medicine and court imagery, ubiquitous throughout the whole dialogue, it will be argued that, far from being an illustration of failure, the text instead indicates that Socrates' conversation with Callicles is successful - and thus proves the efficacy of the elenctic method even on reluctant opponents.
\end{abstract}

\section{Keywords}

elenchus; rhetoric; dialectic; persuasion; understanding; recalcitrance; philosophical life; medicine; punishment 
Es gibt nicht viele Texte, die dem Verstehen so viele Rätsel aufgeben wie die Platonischen Dialoge. Wenn man sich die unterschiedlichen Interpretationen vor Augen hält, die nebeneinander geboten werden, dann kann man es zuweilen kaum glauben, dass vom selben Text noch die Rede sei. Der Gorgias bildet hinsichtlich der Vielstimmigkeit seiner Interpreten beileibe keine Ausnahme. Mein Interesse zielt jedoch auf einen Punkt, über den weitgehend Einigkeit zu bestehen scheint. Im Allgemeinen gilt als ausgemacht, dass es im Dialog dem Protagonisten Sokrates in vielen Anläufen nicht gelingt, seinen Gesprächspartner Kallikles von der philosophischen Lebensführung zu überzeugen.

Bevor ich mich jedoch näher mit der Frage beschäftige, wie viel Sokrates zu überzeugen vermag, sei die Handlung des Dialogs noch einmal kurz rekapituliert: Der Gorgias enthält inhaltlich aufeinander aufbauende Gespräche zwischen Sokrates und den drei Gesprächspartnern Gorgias aus Leontinoi, der Lichtgestalt der Rhetorik schlechthin, Polos, einem seiner Schüler und Kallikles, einem politisch ambitionierten jungen Athener, wobei die Diskussion zunehmend an Schärfe gewinnt. Thema der Gespräche ist eigentlich und zunächst die Rhetorik, die unter den gezielten Fragen des Sokrates recht schnell in ein moralisch bedenkliches Licht gerät, jedem Zweck dienstbar zu sein. In der Folge werden dann allgemeinere ethische Fragestellungen aufgeworfen, etwa ob es sich empfiehlt, Unrecht zu begehen oder sich der legitimen Strafe zu entziehen, bis schließlich in eine Erörterung der alles übergreifenden Frage eingetreten wird, wie man leben soll. Besondere Bekanntheit hat darin die feurige Rede des Kallikles erlangt, in welcher er das natürliche Recht des Stärkeren propagiert, seine Begierden schrankenlos auszuleben.

Neben Gorgias, der als Titelfigur erstaunlich blass bleibt, und Polos, der vor allem durch jugendlichen Übereifer auffällt, entpuppt sich Kallikles als die eigentliche Herausforderung für Sokrates. Nicht von ungefähr nimmt das Gespräch mit ihm mehr Raum ein als die Gespräche mit Gorgias und Polos zusammengenommen. Die besondere Bedeutung, die Kallikles innerhalb des Dialogs innehat, ist auch daran abzulesen, dass der Dialog mit den Worten des Kallikles eröffnet wird und mit der Anrede seines Namens endet. Anfang und Ende, die exponiertesten Partien eines jeden Textes, wurden so für Kallikles reserviert.

Der Disput, den Kallikles und Sokrates im dritten und finalen Akt des Dialogs führen, verläuft zunächst ganz so, wie zu erwarten: Kallikles ist Sokrates argumentativ hoffnungslos unterlegen, er gerät trotz vielerlei Ausflüchte zunehmend in Bedrängnis, auch weil er sich zu immer waghalsigeren Behauptungen versteigt. ${ }^{1}$ Irgendwann ist der Verdruss so groß, dass er für eine gewisse Zeit sogar ganz aus dem Gespräch aussteigt und Sokrates das Feld allein überlässt. Im weiteren Verlauf zeigt er an der Sokratischen Argumentation allenfalls noch mäßiges Interesse. Kallikles ist in der Sache geschlagen, daran besteht nach der Darstellung kein Zweifel, aber er hinterlässt zugleich den Eindruck, als ob ihn das nicht sonderlich tangieren würde. Als ihm Sokrates kurz vor Ende des Zwiegesprächs eine letzte Begründung anbietet, warum er selbst um den Preis des Todes stets

1 Klosko (1984) vermutet, Platon habe unfairerweise dafür gesorgt, dass Sokrates Kallikles leicht widerlegen konnte, indem er diesen seine ursprüngliche Position ohne Not zugunsten einer nicht zu verteidigenden Behauptung preisgeben lässt. 
danach trachten werde, kein Unrecht zu begehen, da gibt Kallikles beinahe gelangweilt zu Protokoll: „Gut, da du auch das andere zu Ende gedacht hast, bringe auch dieses zu Ende. “2 Es ist dies wohlgemerkt Kallikles' letztes Statement im Dialog. Platon scheint in der Tat den Anschein erwecken zu wollen, als sei es Sokrates trotz der Widerlegungen nicht gelungen, Kallikles zur Einsicht zu bringen. Dies ist zumindest die Ansicht der allermeisten Interpreten: Kallikles ist argumentativ überwunden und verharrt dennoch weiter in Opposition zur Sokratischen Sichtweise.

Dieser Befund ist durchaus delikat. Er steht nämlich am Ende eines Dialogs, in dem nicht nur eine substantielle Kritik an der klassischen und rein instrumentell aufgefassten Rhetorik vorgetragen wird. Platon nutzt die Diskussionen auch, um theoretisch und in der praktischen Anwendung allmählich ein positives Gegenstück zur konventionellen Rhetorik aufscheinen zu lassen, welches in der Elenktik bzw. in der philosophischen Dialektik besteht. Dabei soll der Beweis für die Überlegenheit dieser besonderen Gesprächsweise erbracht werden. Der Vorzug der elenktischen Methode erschöpft sich nicht allein darin, dass sie anders als der Relativismus der Rhetorik ganz und gar ethisch-pädagogischen Zwecken verpflichtet ist. Ihr Einsatz soll sich vor allem auch deshalb empfehlen, weil die Gesprächspartner mit ihr zu Zugeständnissen gebracht werden können, die in Widerspruch zu ihren ursprünglichen Überzeugungen stehen. Der elenktische Schluss soll gegenüber dem rhetorischen gewissermaßen eine nachhaltigere Wirkung ausüben. Dies wird bereits im Gespräch mit Polos von Sokrates demonstriert. Nachdem es diesem nicht gelungen ist, Sokrates mit den Mitteln der Rhetorik von seiner Position zu überzeugen, kündigt ihm Sokrates eine Widerlegung an, wie sie sein muss, ${ }^{3}$ und gelangt damit im Gegensatz zu Polos tatsächlich ans Ziel: Polos muss seinen Standpunkt bald aufgeben.

Platon begnügt sich jedoch nicht damit, die Wirkmächtigkeit der Frage- und Antwort-Methode über den praktischen Vollzug manifest werden zu lassen. Der Anspruch auf Überlegenheit wird zusätzlich semantisch aufgeladen, indem die elenktische Widerlegung fortlaufend zunächst mit einer medizinischen Behandlung, später auch mit einer richterlichen Bestrafung verglichen wird, die in ihrer Wirkung zudem annähernd einander gleichgestellt werden. Für beide Verfahren gilt, dass sie zwar schmerzhaft, aber heilsam sind. „Vertraue dich freimütig bei der Antwort dem Logos wie einem Arzt an“, redet Sokrates Polos Mut zu und bringt damit auf den Punkt, was über eine Vielzahl von Beispielen aus der Medizin im Dialog präsent ist. Auf die Parallelisierung der dialektischen Prüfung mit der Bestrafung wurde vor allem in jüngerer Zeit in der englischsprachigen Forschung wieder verstärkt aufmerksam gemacht. ${ }^{5}$ Den Schlüssel für das

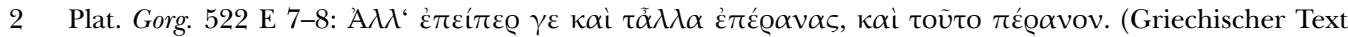
nach der Oxfordgesamtausgabe von J. Burnet). Die Übersetzungen sind sämtlich Erler (2011) entnommen.

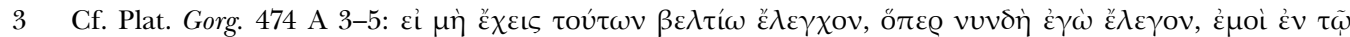

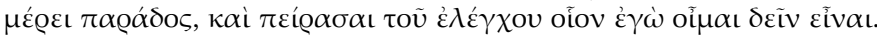

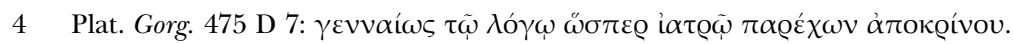

5 Nach Sedley (2009) und Rowe (2007: pp. 148 et seqq.) zielt die Darstellung im Gorgias darauf, mit der Dialektik eine Form der Bestrafung zu präsentieren, mit der die Defizite der herkömmlichen Strafpraxis 
Verständnis dieser Metapher bietet erst der von Sokrates am Ende des Dialogs gebotene Mythos vom Unterweltsgericht, wo den von ihren weltlichen Verfehlungen entstellten Seelen die angemessenen und nutzbringenden Strafen auferlegt werden. Die Verschränkung von Gerichts- und Medizinmetaphorik ist in ihrer Tendenz eindeutig. Es gehört zum Selbstverständnis der wahren Rhetorik, wie die Dialektik beiläufig bezeichnet wird, ${ }^{6}$ korrigierend und bessernd auf die Seelen der Menschen einzuwirken.

Wenn wir nun in Ansatz bringen, wie viel Gewicht auf die Überlegenheit der dialektischen Widerlegung im gesamten Dialog gelegt wird, dann wird, hoffe ich, verständlich, wieso die Widerspenstigkeit des Kallikles nicht so ohne weiteres auf sich beruhen kann. Es bestände eine Spannung zwischen dem mit besonderem Nachdruck vertretenen methodologischen Anspruch und dem tatsächlichen Dialogverlauf. Sollte es tatsächlich Platons Absicht gewesen sein, durch das kallikleische Gesprächsverhalten die Wirkungslosigkeit der Sokratischen Gesprächsweise auf performativer Ebene zur Darstellung zu bringen, würde dies die Ergebnisse der argumentativen Ebene und mithin eine der Hauptaussagen des Textes zunichtemachen. Dies wäre zumal für Platon ungewöhnlich, der auf Stimmigkeit und Verschränkung beider Ebenen stets besonders viel Wert legt.

Zu Kallikles' Uneinsichtigkeit existieren verschiedene Erklärungsansätze. Im Grunde lassen sich zwei Tendenzen in der Forschung beobachten. Es gibt eine Minderheit, nach der die Uneinsichtigkeit tatsächlich auf Unzulänglichkeiten der elenktischen Methode zurückzuführen sei, was eine kritische Distanzierung Platons von der Sokratischen Form der Dialektik nahelege. Der Dialog bezeuge vielmehr ein ernsthaftes Interesse Platons an der Rhetorik. Platon habe im Gorgias zeigen wollen, dass die Dialektik nur dann erfolgreich sein könne, wenn sie auf die Mittel der Rhetorik zurückgreife. Nur die Verbindung beider Techniken könne eine wirkliche Überzeugung der Gesprächspartner herbeiführen. ${ }^{7}$ Diese Literaturmeinungen vermögen trotz einiger hilfreicher Einzelbeobachtungen kaum zu überzeugen. Der Kontrast von zeitgenössischer Rhetorik und Dialektik fällt im Dialog zu dezidiert aus, als dass man das Ziel einer Kombination der beiden Disziplinen für wahrscheinlich halten könnte. In der Mehrzahl wird daher der Grund, dass Sokrates mit seiner rationalen Argumentationsweise letztlich erfolglos bleibe, an der Person des Kallikles festgemacht. Stellvertretend hierfür sei Dominic Scott zitiert: „Plato uses the dialogue form precisely to show the ineffectiveness of philosophical dialogue on a certain kind of interlocutor" ${ }^{8}{ }^{8}$ Theo Kobusch geht noch einen Schritt

überwunden werden. Edmonds (2012) sieht in der Parallelisierung dagegen vor allem ein Mittel, um die Wirkung der Sokratischen Elenktik auf die Gesprächspartner zu illustrieren.

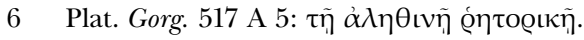

7 Cf. Tarnopolsky (2010: p. 140), McCoy (2009: p. 85 et seqq.) und Stauffer (2006), der den Versuch unternommen hat, mit diesem Ansatz den gesamten Dialog einer Neuinterpretation zu unterziehen. Er greift darin ähnlich gelagerte Überlegungen von Nichols (1998), Kastely (1991) und Black (1958) auf. In die gleiche Richtung haben zuvor auch Renaud (2001) und Kauffman (1979) argumentiert.

8 Scott (1999: p. 25). Bereits Dodds (1959: p. 352 ad 513 c 5) hält es für wahrscheinlich, dass Platon zu erkennen geben wollte, „basic moral attitudes“ seien primär psychisch gegründet und daher rationaler Argumentation nicht zugänglich. Dieselbe Einschätzung vertreten mit Akzentuierungen auch Beversluis (2000: 369 et seqq.) und Dalfen (2004: p. 466). Mit Blick auf die Überzeugung des Kallikles ähnlich skeptisch, hinsichtlich der rationalen Einflussmöglichkeiten insgesamt aber zuversichtlicher urteilen Irwin 
weiter. Er zeigt sich überzeugt, dass Kallikles „für Platon das Sinnbild des Bösen“ darstelle und als „Tyrann des Gesprächs“ gezeichnet werde, da er sich weigere, den stärkeren Logos als solchen anzuerkennen. ${ }^{9}$ Zuletzt rechnete Radcliffe Edmonds Kallikles zu den Unheilbaren, auf die Sokrates im bereits genannten Schlussmythos zu sprechen kommt. Darin stellt Sokrates fest, dass die ärgsten Übeltäter sich selbst um die Möglichkeit bringen können, durch die Bestrafung an der Seele kuriert zu werden. Gleichwohl zu ewiger Bestrafung verdammt, dienten sie den anderen Menschen als warnende Beispiele, ein Schicksal, das besonders Königen und Tyrannen drohe. ${ }^{10}$ Nach Edmonds ist Kallikles aufgrund seiner beständigen Weigerung, die Widerlegungen anzuerkennen, ebenfalls zu dieser traurigen Gruppe zu zählen. ${ }^{11}$

Die Interpretationen, welche die Figur des Kallikles in Haftung nehmen, haben den Vorteil, dass sie den Primat der philosophischen Dialektik unangetastet lassen. Es ist jedoch festzustellen, dass sie die Spannung, die ich eingangs beschrieben habe, nicht eigentlich auflösen. Die philosophische Methode wird zwar gegen den Verdacht der Untauglichkeit in Schutz genommen. Es bleibt dennoch merkwürdig, dass der Leser, der ja von der Leistungsfähigkeit der Dialektik überzeugt werden soll, gerade zum Ende des Dialogs hin mit ihren Grenzen konfrontiert wird. Die beabsichtigte Wirkung auf den Leser, so wir sie denn richtig bestimmt haben, würde zumindest deutlich abgeschwächt.

Im Folgenden soll daher der Versuch unternommen werden zu zeigen, dass im Gorgias der Sokratischen Elenktik eine größere Wirkung auf Kallikles zuzuschreiben ist, als im Allgemeinen angenommen wird. Es ist zwar einerseits richtig, dass Kallikles nicht vollauf von der philosophischen Lebensführung überzeugt wird. Andererseits enthält der Dialog Aspekte, die dem Leser signalisieren, dass das Gespräch mit Sokrates an Kallikles nicht spurlos vorübergeht, sondern dass dieser vielmehr in einem Maße beeindruckt wird, wie es zur Grundintention des Textes passt.

Zunächst einmal finden sich im Text einzelne Gesichtspunkte, die nicht ohne Weiteres mit der gängigen Lesart in Einklang zu bringen sind und die eher darauf hindeuten, dass wir das Sokratische Vorgehen auch Kallikles gegenüber als wirksam betrachten sollen. Gesetzt den Fall, es wäre die Absicht des Autors gewesen, Kallikles als Negativbeispiel vorzuführen, dem auch mit den Mitteln der Dialektik nicht beizukommen ist, dann bliebe die Frage zu beantworten, mit welcher Motivation er Sokrates das Gespräch auf der internen Dialogebene führen lässt. Es spräche nicht gerade für Sokrates' dialektischen Sachverstand, wenn wir annähmen, er komme erst am Ende des Gesprächs zu der Einsicht, dass alle Mühe vergeblich gewesen sei. Daher müsste man davon ausgehen, dass auch er sich von Beginn an über die Aussichtslosigkeit des Unterfangens im

(1979: p. 233), Woolf (2000) und Pedrique (2011: pp. 243 et seqq.). Anders jedoch McKim (1988), Blank (1993), Moss (2005) und Collobert (2013), die das Hauptziel der Sokratischen Gesprächsführung auf je unterschiedliche Weise in einer psychologischen Manipulation der Gesprächspartner sehen. Abwegig ist schließlich die These von Arieti (1993), nach der Kallikles unter den Händen von Sokrates sogar moralisch degeneriere.

9 Kobusch (1978: p. 101).

10 Cf. Plat. Gorg. 525 D 2-E 2.

11 Edmonds (2012: p. 178). 
Klaren ist, Kallikles zu überzeugen. ${ }^{12}$ Dies dürfte aber angesichts der großen Geduld, die Sokrates für seinen Gesprächspartner aufbringt, wenig wahrscheinlich sein. Eine solche Annahme stände auch in einer merkwürdigen Spannung zum Schlussplädoyer, mit dem Sokrates Kallikles ins Gewissen redet, sich wieder auf den Weg des Gesetzes zu begeben. Sokrates wendet sich in seinen Worten ausdrücklich an die Person des Kallikles:

„Lass dich also von mir überzeugen und folge dorthin, wo du, wenn du ankommst, glücklich sein wirst im Leben und im Tod, wie die Geschichte zeigt. “13

Es ist schwer vorstellbar, dass Sokrates derart eindringliche Worte an Kallikles richtet, ohne Hoffnung zu haben, ihn zu erreichen. Sowohl die Länge des Gesprächs als auch die Ansprache zum Schluss lassen daher auf einen größeren Optimismus hinsichtlich der Wirkung der Sokratischen Reden schließen.

Es verwundert zudem auch, dass der junge Kallikles Tyrannen als den schlimmsten Übeltätern gleichgestellt sein soll, die ein ganzes Leben lang Verbrechen an Verbrechen gereiht haben und die davon an der Seele ungeheure Missbildungen davongetragen haben. Im Dialog wird hingegen mehrfach darauf hingewiesen, dass Kallikles noch ganz am Anfang seiner Karriere stehe. ${ }^{14}$ Warum sollte bei ihm schon zu diesem frühen Zeitpunkt alles verloren sein? Auch in diesem Punkt scheint die Darstellung bereits von einer größeren Zuversicht bestimmt zu sein, als man zunächst anzunehmen geneigt ist.

Wie gehen wir nun aber mit den zahlreichen ablehnenden Reaktionen des Kallikles um? Scheinen sie nicht eine eindeutige Sprache zu sprechen? Ein ums andere Mal tut Kallikles die Resultate der gemeinsamen Untersuchung als dummes Zeug ab. Mal mockiert er sich süffisant, mal gibt er seinem angeblichen Unverständnis Ausdruck. ${ }^{15}$ Deutlicher lässt sich eine Distanz kaum ausdrücken, so scheint es. Um die Reaktionen jedoch richtig einzuordnen, empfiehlt sich von neuem ein Blick auf die begleitende Bildsprache des Dialogs.

Radcliffe Edmonds hat zunächst richtig gesehen, dass die Gerichts- und Medizinmetaphorik, die Platon im Dialog nachgerade profiliert, der Illustration der Effekte dient, die eine dialektische Widerlegung beim Antwortenden hervorruft. ${ }^{16}$ Doch anders als Edmonds annimmt, kann sich derjenige, der sich einer dialektischen Prüfung unterwirft, der Wirkung, die durch die Widerlegung hervorgerufen wird und die einer ärztlichen Operation oder einer gerichtlichen Bestrafung vergleichbar ist, gar nicht entziehen. ${ }^{17}$

12 Nach Ansicht von Lewis (1986) steht es nicht in Sokrates' Absicht, Kallikles zu überzeugen, sondern lediglich das anwesende Publikum für sich zu gewinnen.

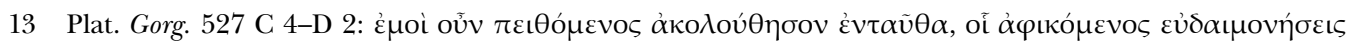

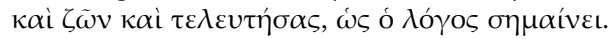

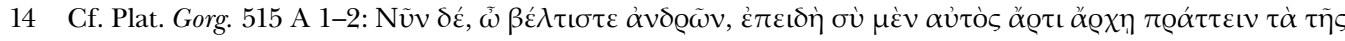
$\pi 0 ́ \lambda \varepsilon \omega \varsigma \pi \varrho \alpha ́ \gamma \mu \alpha \tau \alpha$.

15 Cf. z.B. die Reaktionen des Kallikles Plat. Gorg. 489 B 7-C 1; 490 E 4; 494 A 6; 497 A 6.

16 Edmonds (2012: p. 166): ,the description of the judgement and punishment as the examination and healing of a soul scarred with wounds and disease illuminates the effects of the elenchos on the interlocutors".

17 Cf. Edmonds (2012: p. 177). 
Ist die Operation einmal durchgeführt und ist die Bestrafung einmal vollzogen, können die Auswirkungen auf den Betroffenen nicht mehr ungeschehen gemacht werden. Legt man darum die Parallele zu den beiden Lebensbereichen konsequent aus, dann ist die beabsichtigte korrektive Erfahrung im Moment der Widerlegung selbst zu denken. Ein nachträgliches Abrücken vom Ergebnis der Prüfung dürfte an der Wirksamkeit - nimmt man die Gleichsetzung ernst - an sich nichts ändern. Kallikles' permanente Distanzierungsversuche scheinen daher etwas anderes anzuzeigen. Den Schlüssel für das Verständnis bietet ein Aspekt, auf den bereits im Laufe des Dialogs wiederholt und zuletzt im Schlussmythos von Sokrates hingewiesen wird. Wer heilbare Verfehlungen begangen hat, kann nur unter Schmerzen kuriert werden. ${ }^{18}$ Was für die Strafe gilt, hat gemäß der Analogie auch für die Widerlegung zu gelten. Sie ist zwar heilsam, zugleich aber notwendig unangenehm und schmerzhaft. Richtet man sich strikt nach der Logik des Vergleichs müssen wir auch für Kallikles annehmen, dass er von diesen unangenehmen Begleiterscheinungen nicht verschont bleibt. Wie aber macht man ein akutes Schmerzempfinden in einer dramatischen Handlung sichtbar? Könnte es nicht sein, dass die verbalen Angriffe, die Kallikles regelmäßig gegen Sokrates vorträgt, als Reflexe auf Sokrates' schmerzhafte Interventionen zu interpretieren sind, als Ausweis einer emotionalen Not, in die er durch die Argumentation gebracht wird? Eine derartige Figurenmotivation entspräche durchaus allgemeiner Erfahrung, denn ein erzwungenes unangenehmes Eingeständnis ruft nicht selten heftige und unverhältnismäßige Aggressionen hervor. Wenn dies richtig ist, dann stehen Kallikles' Vorhaltungen somit nicht für die Wirkungslosigkeit, sondern im Gegenteil gerade für die Wirksamkeit der dialektischen Gesprächsmethode. ${ }^{19}$ Kallikles ist sehr wohl angefasst und gibt sich Mühe, der unangenehmen Gesprächssituation zu entkommen. Sein Gesprächsverhalten ist in erster Linie durch Ablenkungsmanöver gekennzeichnet, mit denen er zudem versucht, sein Gesicht zu wahren. Wie zur Bestätigung fallen die Abwehrreaktionen im unmittelbaren Anschluss an eine erfolgreiche conclusio, die sich Kallikles aufgrund seiner vorherigen Zustimmungen zurechnen lassen muss, stets besonders vehement aus. ${ }^{20}$ In derselben Weise ließe sich auch erklären, warum er zuweilen den Eindruck macht, als nehme er an der Unterredung mit Sokrates kaum mehr Anteil. Da Kallikles offenbar den Hang hat, seine Hilflosigkeit zu kaschieren, kann das Desinteresse auch gespielt sein.

Ein weiteres Indiz für eine erfolgreich zu denkende Intervention scheint mir darin zu liegen, dass man bei Kallikles mit fortschreitender Entwicklung ein allmähliches Abflauen der Emotionen registrieren kann. Kallikles steigt mit ungeheurem Impetus in die Unterredung ein und schwingt sich auch in der Folge immer wieder zu heftigen Reden auf. Die Schlagkraft schwindet jedoch zusehends. Nachdem er Sokrates auch durch seinen vorübergehenden Gesprächsboykott nicht zum Schweigen bringen konnte, erleben wir ein letztes Aufbäumen bereits weit vor Ende des Gesprächs. ${ }^{21}$ Er verzichtet auch

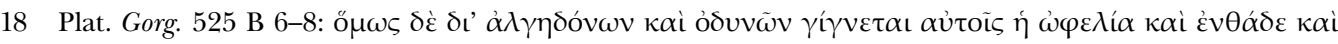
$\varepsilon \dot{\varepsilon} \mathrm{A}$ Atoov.

19 Cf. hingegen Scott (1999: p. 25).

20 Cf. Plat. Gorg. 489 B 7-C 7; 494 E 7-8; 497 A 6; 499 B 4-8; 505 C 1-2; 511 A 4-7.

21 Plat. Gorg. 511 A 4-7. 
danach nicht auf Protest, dieser bleibt aber deutlich unter dem anfänglichen Erregungsniveau. Wie die Beobachtungen zeigen, bietet der Text eine Reihe von Anhaltspunkten, die es wahrscheinlich machen, dass Kallikles von Sokrates doch stärker affiziert wird, als es zunächst scheinen mag.

Doch nicht nur die emotionale Erregung ist auffällig. Wie ich im Folgenden ausführen werde, lässt sich zudem beobachten, dass sich auch Kallikles' theoretische Positionen unter dem Einfluss der Sokratischen Argumentation sukzessive verändern: Kallikles kombiniert in seiner flammenden Rede zu Beginn zwei Thesen, die bei genauem Hinsehen in einem Spannungsverhältnis zueinander stehen. Zum einen stellt er eine Art Herrenmoral auf, nach der von Natur aus allein gerecht ist, was dem Stärkeren gut scheint. Zum anderen verachtet er diejenigen, die unvermögend sind, sich gegen Unrecht zur Wehr zu setzen. ${ }^{22}$ Beide Behauptungen sind Ausdruck eines elitären Selbstverständnisses, das auch in der weiteren Diskussion immer wieder Thema ist. ${ }^{23}$ Sokrates referiert Kallikles' Standpunkt beim Einstieg in die gemeinsame Prüfung wie folgt:

„Dass der Stärkere mit Gewalt das wegführt, was den Schwächeren gehört, und dass der Bessere über den Schlechteren herrscht und dass der Tüchtigere mehr hat als der weniger Tüchtige?" 24

Von Sokrates im Handumdrehen argumentativ unter Druck gesetzt, ist Kallikles bald gezwungen, seine These vom Recht des Stärkeren zu retten, indem er sie in die Forderung ummünzt, seine Begierden schrankenlos auszuleben. ${ }^{25}$ Spätestens hier ist Kallikles unwiederbringlich verloren, denn in dieser extremen Ausprägung lässt sich seine Ausgangsthese unter keinen Umständen halten, am allerwenigsten aber gegenüber einem dialektisch geschulten Gesprächspartner wie Sokrates. ${ }^{26}$ Kallikles wehrt sich zwar nach Kräften, am Ende muss aber auch er zugeben, dass das Gute und das Angenehme nicht gleichzusetzen sind und es daher nicht erstrebenswert sein kann, allen Begierden gleichermaßen zu frönen. Wie Jessica Moss jüngst richtig herausgestellt hat, gelingt es Sokrates also bereits in einem ersten entscheidenden Schritt, Kallikles von einer grundlegenden Überzeugung abzubringen. ${ }^{27}$ Kallikles distanziert sich tatsächlich expressis verbis von seiner ursprünglichen Position. Es ist indes entlarvend, auf welche Weise er am Ende einlenkt:

22 Plat. Gorg. 483 A 5-486 C 3. Ober (1998: p. 198 et seqq.) hat in seiner gründlichen Analyse der Rede die Widersprüchlichkeit der Gedankenführung eindrucksvoll herausgearbeitet.

23 Cf. z.B. Plat. Gorg. 491 A 1-3; 512 C 3-D 2.

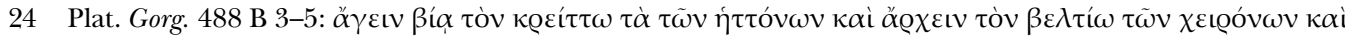

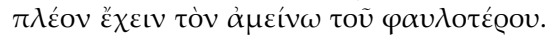

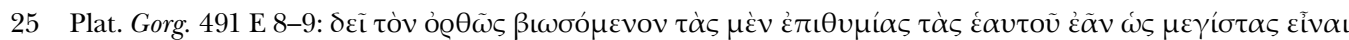

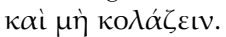

26 Cf. Klosko (1984).

27 Moss (2005: p. 150). Dagegen vertritt Beversluis (2000: 349) die Auffassung, dass es nie Kallikles' Überzeugung gewesen sei, alle Begierden schrankenlos auszuleben; die Behauptung sei ihm von Sokrates geschickt untergeschoben worden. 
„Lange schon höre ich dir zu, Sokrates, und stimme dir immer wieder zu, weil ich denke, dass du wie die Kinder gerne daran festhältst, wenn einer dir im Scherz etwas zugibt. So bist du offenbar wirklich der Meinung, dass ich oder ein anderer Mensch nicht der Meinung sei, dass es bessere und schlechtere Genüsse gibt. “28

Es sei daran erinnert, dass es sich um eine seiner zentralen Überzeugungen handelte. Kallikles gibt sie preis, als hätte ihm nie daran gelegen gewesen. Augenscheinlich hindert ihn die Scham, die er ob der endgültigen Widerlegung empfindet, auch die Niederlage als solche offen anzuerkennen.

In einem Punkt zeigt sich Kallikles freilich auch weiterhin uneinsichtig. Sooft Sokrates darangeht, ihn von der Notwendigkeit der Selbstbeherrschung zu überzeugen, verweigert er sich rigoros. Es ist eben diese Schlussfolgerung, die Kallikles dazu veranlasst, aus dem Gespräch auszubrechen. ${ }^{29}$ Wie der Tadel an Sokrates' philosophischer Lebensführung verrät, stellt für Kallikles allein die Vorstellung, einer ungerechten Behandlung hilflos ausgeliefert zu sein, eine Beleidigung für sein sublimes Ehrgefühl dar. Selbstbeherrschung kommt für ihn daher gewissermaßen einem freiwilligen Verzicht auf die angestammten Privilegien und einem vorauseilenden Gehorsam gleich. ${ }^{30}$ Aufgrund der Tatsache, dass das Thema bei Kallikles offenkundig einem Tabu unterliegt, ist Sokrates zu einem Umweg gezwungen. Er muss sich zunächst auf die Perspektive des Kallikles einlassen, indem er der Frage nachgeht, mit welchen Mitteln man wirksam verhindern kann, Unrecht zu erleiden. ${ }^{31}$ Gleichzeitig verfolgt er damit die verdeckte Strategie, Kallikles mit seinem elitären Selbstverständnis in Konflikt zu bringen. Wie bei der Bekämpfung der Hedonismusthese agiert Sokrates offenbar auch hier nicht ohne Erfolg. Sokrates' Räsonnement, einzig die Anpassung an die Herrschenden biete einen wirksamen Schutz vor Unbill, leuchtet Kallikles so unmittelbar ein, dass er seine Zustimmung in ungewohnt bereitwilliger Form erteilt, auch wenn er grundsätzlich skeptisch bleibt. ${ }^{32}$ Erst kurz vor Schluss führt Sokrates dann den entscheidenden Stoß, indem er Kallikles vor die Alternative zwischen den beiden konkurrierenden Lebensformen stellt, deren Vergleich die gemeinsame Untersuchung gedient hat. Betrachten wir den Wortwechsel im Detail!

„Sokrates: Leg mir fest, zu welchem Dienst für die Stadt du mich aufforderst: Mit den Athenern durchzukämpfen, dass sie möglichst gut werden, wie ein Arzt oder wie einer, der sich ihnen andienen und nach dem Munde reden will? [...] Sprich also auch jetzt schön und freimütig ( $\gamma \varepsilon v v \alpha i ́ \omega \varsigma)$. Kallikles: Ich sage also, wie einer, der sich andienen will. Sokrates: Du rufst

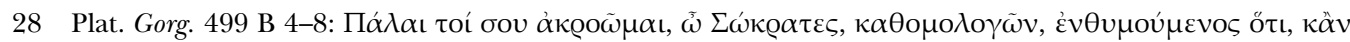

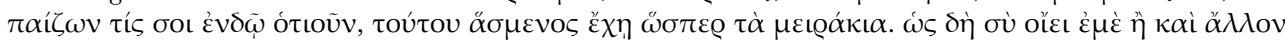

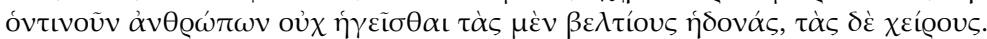

29 Cf. Plat. Gorg. 505 B 11-C 2.

30 Cf. Plat. Gorg. 491 D 9-E 2.

31 Cf. Plat. Gorg. 509 C 6 et seqq.

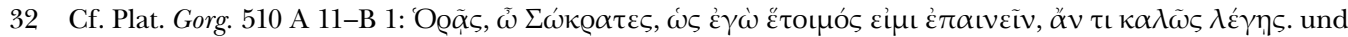

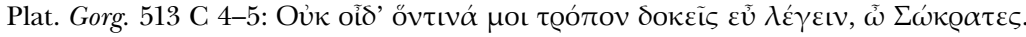




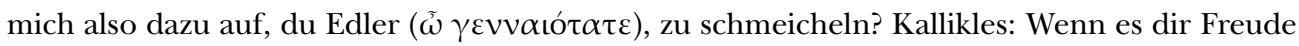
macht, mich einen Myser zu nennen, Sokrates.“33

Wenn Kallikles hier die zweite Option wählt, hat es zunächst den Anschein, Sokrates' Bemühen wäre umsonst gewesen: Kallikles konnte nicht für den philosophischen Lebensweg gewonnen werden. Wer aber glaubt, Kallikles sei dabei seiner Überzeugung treu geblieben, der irrt. Kallikles hat sich von seiner ursprünglichen Position so weit entfernt wie irgend möglich! Dies wird deutlich, wenn wir sein Statement mit seiner Ausgangsposition vergleichen. Kallikles, der noch zu Beginn in einem Anflug von Hochgefühl postulierte, der Stärkere müsse über den Schwächeren herrschen und dürfe sich nicht zum Sklaven einer minderwertigen Moral machen lassen, ${ }^{34}$ scheint es nun klug, sich unterzuordnen und anzudienen! Wie viel ist hier noch von seiner ursprünglichen Herrenmoral geblieben? Sokrates ist es offenbar gelungen, ihn vor sich selbst gründlich $\mathrm{zu}$ blamieren und ihm die Konsequenzen seiner Lebensausrichtung in aller Deutlichkeit ins Bewusstsein zu bringen. Er hat gezeigt, dass Gorgias' Rhetorik der Anpassung, von der sich Kallikles Unabhängigkeit versprach, direkt in die Abhängigkeit führt. Und was noch schlimmer ist: Kallikles wusste sich nicht anders zu helfen, als ihm dabei zu sekundieren. Alles, was er im Anschluss noch an Einwänden vorzubringen weiß, wirkt im Vergleich zum prätentiösen Einstieg müde und hoffnungslos. Wieder läuft Kallikles nicht mit wehenden Fahnen über, aber er ist benommen und angezählt.

Der Ehrverlust ist von Sokrates wohl kalkuliert und zusätzlich im Text markiert. Sokrates ermahnt Kallikles ausdrücklich, so zu sprechen, wie es seiner vornehmen Abstammung geziemt (521 A 7: $\gamma \varepsilon v v \alpha i ́ \omega \varsigma)$, und damit uns die Diskrepanz zum ursprünglichen elitären Selbstgefühl nicht entgeht, setzt Sokrates dies auch bei seiner Rückversicherung in der Anrede noch einmal hinzu (521 B 1: $\tilde{\omega} \gamma \varepsilon v v \alpha \iota$ ó $\alpha \tau \varepsilon)$. Diese Vorgehensweise verfehlt schließlich auch beim Adressaten der Worte ihre Wirkung nicht. Wie Kallikles in seiner Replik zu erkennen gibt, ist er sich des Entwürdigenden seiner Wahl durchaus bewusst. Er scheut sich jedenfalls nicht, das Kind beim Namen zu nennen, indem er mit dem Myser eine sprichwörtlich verachtenswerte Existenz zum Vergleich heranzieht. ${ }^{35}$ Wir dürfen uns also sicher sein, dass Kallikles in einen schmerzhaften Selbstwiderspruch geführt worden ist, der ihm zu denken geben wird. ${ }^{36}$

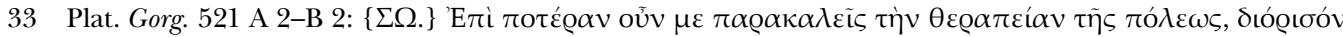

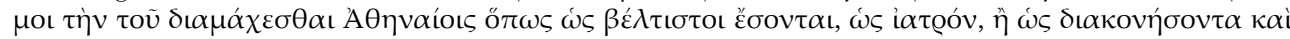

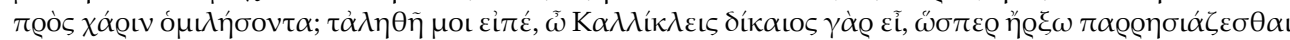

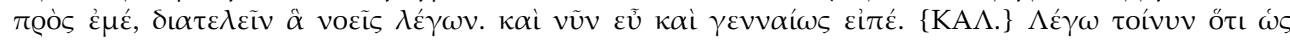

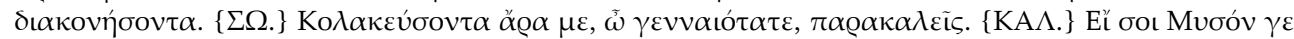

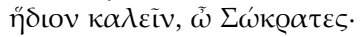

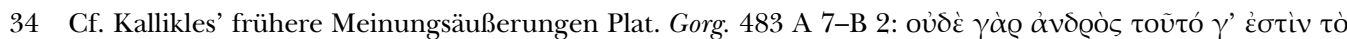

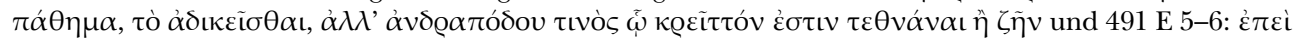

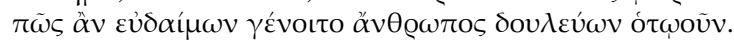

35 Cf. Dalfen (2004: p. 475 ad 521 b).

36 Die hier vorgetragene Interpretation berührt sich in einigen Punkten mit der von Babut (1992: pp. 83-91), der Sokrates jedoch vornehmlich gegen den Vorwurf in Schutz nehmen möchte, den Sieg gegen Kallikles auf sophistische Art und Weise erzielt zu haben. Für Babut ist das Ziel der refutativen Dialektik von vornherein auf die Befreiung von irrtümlichen Ansichten beschränkt. Im Ergebnis hält er Sokrates' 
Zugegeben, Platon lässt es bei diesen spärlichen Signalen bewenden und man könnte fragen, warum er den Effekt nicht deutlicher markiert und damit Missverständnissen vorgebeugt hat? Wenn man es jedoch genau betrachtet, dann erschiene es unter realistischen Bedingungen wenig plausibel, wenn es Sokrates in einem einzigen Gespräch gelingen sollte, seinen Widersacher ganz und gar zu bekehren. Und es wäre noch weniger plausibel, wenn man die gewaltige Diskrepanz der Ausgangspositionen in Anschlag bringt, die die beiden Opponenten vertreten. ${ }^{37}$ Insofern scheint mir hier eher einmal mehr Platons psychologisches Einfühlungsvermögen und sein besonderes Geschick bei der Charakterzeichnung am Werk.

Nicht zu verachten der Effekt, der durch die Zurückhaltung beim Leser erzielt wird. Mit der Entscheidung des Kallikles bleiben beide Optionen im Spiel. Der Leser wird nicht aus der Verantwortung entlassen, sich selbst ein Urteil zu bilden. Der Dialog ist dadurch nicht didaktisch oder belehrend. Es ist vielmehr durch eine gewisse Offenheit gekennzeichnet, wie sie für die aporetischen Frühdialoge insgesamt typisch ist.

\section{Bibliographie}

Arieti, J. (1993). Plato's Philosophical Antiope. The Gorgias in Plato's Dialogues. In G. Press (Ed.), Plato's Dialogues. New Studies and Interpretations (pp. 197-214). Boston: Roman \& Littlefield.

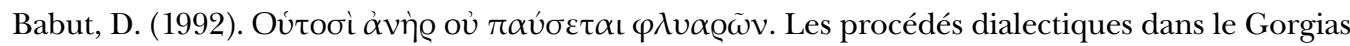
et le dessein du dialogue. Revue des Ėtudes Grecques, 105, 59-110.

Beversluis, J. (2000). Cross-Examining Socrates. A Defense of the Interlocutors in Plato's Early Dialogues. Cambridge: University Press.

Black, E. (1958). Plato's View of Rhetoric. Quarterly Journal of Speech, 43, 361-74.

Blank, D. L. (1993). The Arousal of Emotion in Plato's Dialogues. Classical Quarterly, 43(2), $428-439$.

Collobert, C. (2013). La Rhétorique au Cœur de l'Examen Réfutatif Socratique. Le Jeu des Emotions dans le Gorgias. Phronesis, 58, 107-138.

Dalfen, J. (Transl.). (2004). Platon: Gorgias. In E. Heitsch, \& C. W. Müller (Eds.), Platon: Werke (Bd. VI 3). Göttingen: Vandenhoeck \& Ruprecht.

Dodds, E. (Ed.). (1959). Plato: Gorgias. Oxford: Clarendon Press.

Edmonds, R. (2012). Whip Scars on the Naked Soul. Myth and Elenchos in Plato's Gorgias. In C. Collobert, P. Destrée, \& F. Gonzalez (Eds.), Plato and Myth (pp. 165-185). Leiden - Boston: Brill.

Vorgehen daher für erfolgreich. Ebenso sieht Collobert (2013) in dem Umstand, dass Polos und Kallikles bis zum Schluss nicht bereit sind, Sokrates' Überzeugungen zu übernehmen, kein Defizit der Dialektik. Da Sokrates lediglich darauf aus sei, seinen Gesprächspartnern protreptisch die Notwendigkeit einer philosophischen Wahrheitssuche aufzuzeigen, könne von einem Scheitern keine Rede sein, solange es ihm gelingt - wie im Gorgias der Fall - die ursprünglich vertretenen Positionen logisch zu Fall zu bringen.

37 Cf. Collobert (2013: p. 136): „la remise en question de leur [i.e. Polos und Kallikles] mode de vie est trop radicale et les propositions socratiques trop atopiques pour susciter leur adhésion". 
Erler, M. (Transl.). (2011). Platon: Gorgias (Kommentiert und mit einem Nachwort versehen von T. Kobusch). Stuttgart: Reclam.

Irwin, T. (Transl.). (1979). Plato: Gorgias. Oxford: Clarendon Press.

Kastely, J. (1991). In Defense of Plato's Gorgias. Publications of the Modern Language Association of America, 106, 96-109.

Kauffman, Ch. (1979). Enactment as Argument in the Gorgias. Philosophy and Rhetoric, 12(2), 114-129.

Klosko, G. (1984). The Refutation of Callicles in Plato's 'Gorgias'. Greece E Rome, Second Series, 31(2), 126-139.

Kobusch, Th. (1978). Sprechen und Moral. Überlegungen zum platonischen „Gorgias“. Philosophisches Jahrbuch, 85, 87-108.

Lewis, Th. (1986). Refutative Rhetoric as True Rhetoric in Plato's Gorgias. Interpretation, 14, 195-210.

McCoy, M. (2009). Plato on the Rhetoric of Philosophers and Sophists. Cambridge: University Press.

McKim, R. (1988). Shame and Truth in Plato's Gorgias. In Ch. Griswold (Ed.), Platonic Writings, Platonic Readings (pp. 34-48). New York - London: Routledge.

Moss, J. (2005). Shame, Pleasure, and the Divided Soul. Oxford Studies in Ancient Philosophy, 29, 137-170.

Nichols, J. (1998). The Rhetoric of Justice in Plato's Gorgias. In Idem (Transl.), Plato: Gorgias (pp. 131-149). Ithaca - London: Cornell University Press.

Ober, J. (1998). Political Dissent in Democratic Athens. Princeton: University Press.

Pedrique, N. (2011). Logos dynastes. Dichtung und Rhetorik in Platons Gorgias. Frankfurt am Main: Peter Lang.

Renaud, F. (2001). La rhétorique socratico-platonicienne dans le Gorgias (447a-461b). Philosophie antique, 1, 65-86.

Rowe, Ch. (2007). Plato and the Art of Philosophical Writing. Cambridge: University Press.

Scott, D. (1999). Platonic Pessimism and Moral Education. Oxford Studies in Ancient Philosophy, 17, 15-36.

Sedley, D. (2009). Myth, Punishment and Politics in the Gorgias. In C. Partenie (Ed.), Plato's Myths (pp. 51-76). Cambridge: University Press.

Stauffer, D. (2006). The Unity of Plato's Gorgias. Cambridge: University Press.

Tarnopolsky, Ch. (2010). Prudes, Perverts, and Tyrants. Plato's Gorgias and the Politics of Shame. Princeton: University Press.

Woolf, R. (2000). Callicles and Socrates. Psychic (Dis)Harmony in the Gorgias. Oxford Studies in Ancient Philosophy, 18, 1-40.

Bernhard Kaiser, M.A. / bernhard.kaiser@tu-dresden.de

Institute of Classical Philology

Dresden University of Technology, Faculty of Linguistics, Literature and Cultural Studies

Wiener Str. 48; Raum 404, 01062 Dresden, Germany 